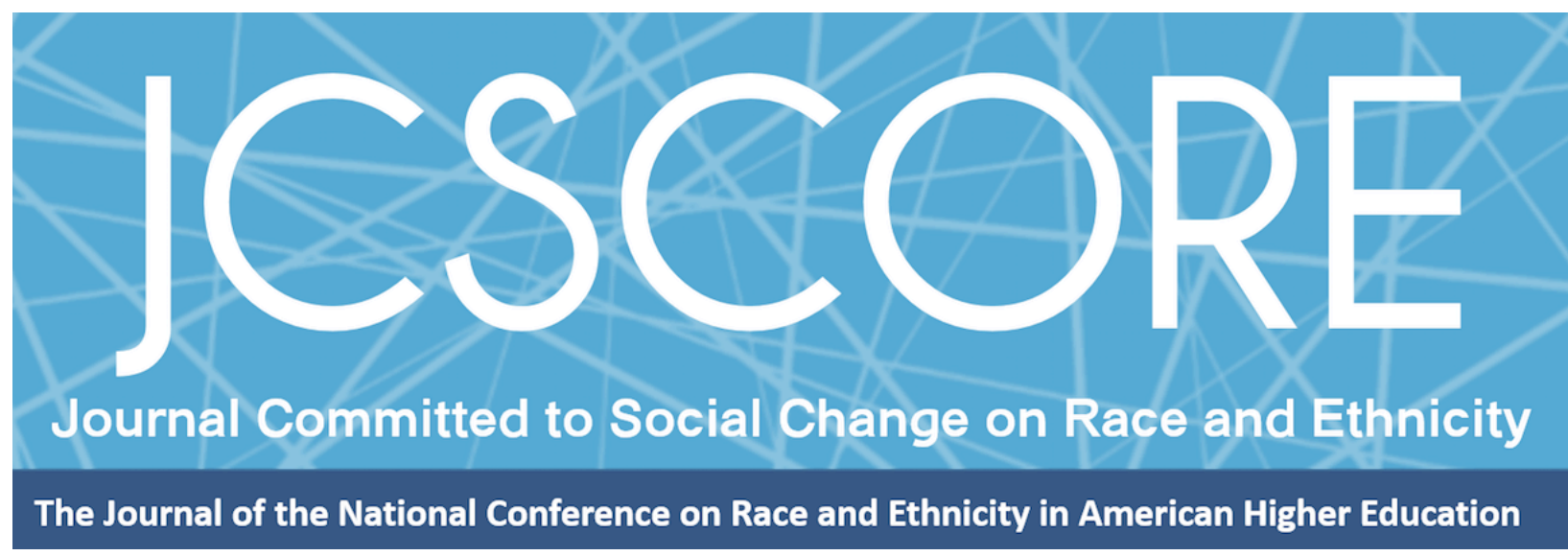

\title{
JUST A UNICORN
}

\author{
Kat J. Stephens \\ University of Massachusetts Amherst
}

Journal Committed to Social Change on Race and Ethnicity

Volume 6, Issue 1 | 2020

\section{Copyright and Open Access}

(C) 2020 Kat J. Stephens

\section{(c) (i) (2)}

This work is licensed under a Creative Commons Attribution-NonCommercial-ShareAlike 4.0 International License. Permission of the authors is required for distribution and for all derivative works, including compilations and translations. Quoting small sections of text is allowed as long as there is appropriate attribution and the article is used for non-commercial purposes.

The Journal Committed to Social Change on Race and Ethnicity (ISSN 2642-2387) is published by the National Conference on Race and Ethnicity (NCORE), a production of the University of Oklahoma, in partnership with the University of Oklahoma Libraries. 


\title{
Just a Unicorn
}

Kat J. Stephens

University of Massachusetts Amherst

\begin{abstract}
Kat J. Stephens is a higher education Ph.D. student at University of Massachusetts Amherst. She's earned a Master of Education from Teachers College, Columbia University, in Higher \& Postsecondary Education. Her larger research interests are social justice \& identity development. As an Afro-Guyanese immigrant, her research interests reflects: Caribbean students, Afro-Caribbean racial identity formation, transnationalism, Black women students with ADHD \& Autism, \& gifted community college \& transfer students. Her work here is inspired by her life and those of other Black women \& girls in educational spaces. This poem serves to highlight her frustrations, while encouraging Black women to take space in disability centered environments, and universities to adequately support such individuals.
\end{abstract}

\section{Just another Model Immigrant}

Dem: Yuh mek yuhself dotish or wah?

Mi: But

Dem: Yuh nuh ave' no dis-ah-bil-uhTEE

Mi: But

Dem: *suck teeth*

Dem: Yuh too smart for alluh dat

\section{Just another Misunderstood Gem}

Reading at Two

Gifted Programs

Skipping Grades

Honor Societies

Gifted kid gone rogue

Wasted Potential

Bored in the classroom

Almost failed out of high school

Gifted college dropout 


\author{
Just another Monday \\ My brain pains me \\ Thick, translucent fog \\ Cradling then choking with overwhelm \\ Professor's words, I cannot follow \\ Fog from my shoulders to my eyeballs, \\ Brows furrowed more deeply with each directionless thought \\ Is this real? \\ Left tilted head; hearing words and sounds that mean nothing \\ Right tilted head; open stance begging and inviting focus to select me \\ $30,40,50$, Minutes into class and my brain finally gets in formation \\ Blue stained fingertips, Adder*ll dust visible \\ Finally note-taking with intention \\ Flick of the wrist-dance, nails tap dancing on my keyboard \\ Just in time for the wind-down of class \\ A jumbled race of questions, thoughts, points of clarity and eagerness \\ My supernaturally molasses brain is now quickened quicksand \\ Emotions on a thousand, contained to my body, chained to this seat
}

\title{
Just another Tuesday
}

Mistake number seventy-five

Deleted another final paper

Three minutes to eleven fifty-nine's deadline

Obsessively reminding my mind to not forget

Only for anxiety and adrenaline to quarrel it out

Sweat bridling down my left temple

Heart racing, making Timbaland-like beats

Yet another begging email being composed

"Dear Dr." insert [Whiteness] here

"Would you please grant" insert [Black] here

"Me an extension" insert [Woman] here

"I do apologize" insert [First-Generation] here

"For the inconvenience" insert [Immigrant] here

\section{Just another life}

What I would give to be typical [neuro]

Somebody's embodiment of diversity [neuro]

Living as a Black woman with ADHD

Learning as a Black woman with ADHD

Zipping through elementary and middle school

Honors student throughout

Around grade nine, I was just \& barely fine

Dipping from honors \& AP to gym for a GPA boost

Secondary school life without a diagnosis 


\section{Just another Life}

Passing high school one point above its minimum

Never did the homework, barely did the readings

Found the most in class, to say

Teachers perplexed day by day

"So much potential" they'd say

School carried the pain of a thousand papercuts

I was bored anyway,

And learning did me nothin' [but harm]

College was a repetitive rubber stamp

Except I didn't have to go to class

So... you could guess I didn't

\section{Just another Urban Fairytale}

My school was poor

My hood far too Black \& Brown

My boredom meant I walked away

There was nothing nobody could say

I loved to learn; I hated school, so

Dropping out saved my life

Because when I finally re-enrolled

It was me and this journey

Public community college

To elite liberal arts

To the Ivy League

To a fully funded doctorate Riddled with imposter syndrome

Familiarity in reading, writing

Forgetfulness along the way

Amplified by fear and tears

\section{Just another differently abled student}

Would they realize

They made a mistake

By admitting me?

When fortitude

Resourcefulness, and creativity aren't enough

This professional student felt $d^{*} m b$, inc ${ }^{*}$ pable, sl ${ }^{*} w$ Internalized ableist language and feelings

Reflected upon myself

My ADHD symptoms internalized as personal defects 


\author{
Just another Ableist University System \\ I'm working twice as hard \\ In neurotypical university spaces \\ That lack universal design \\ Pretending not to see us outside of disability office spaces \\ Where we need supportive accommodations \\ $\mathrm{OH}$ ! the anger, the loss, the relief \\ That came to me in doctoral studies \\ And FINALLY, a doctor said \\ "I think it could be ADHD" \\ I cried tears that were thick and hot \\ At my big age, \\ Decades spinning in barely functioning schoolhouses \\ Where different means push out \\ Where different means jailhouse \\ Because I lived my whole life afraid \\ Internalizing my neuro-diverse self \\ As a br*ken, no-good, intellectually inferi* $r$ \\ Forgetful, careless, dimw ${ }^{\star}$ t self \\ Meanwhile the involuntary hacks \\ Built in the recesses of my brain??? \\ Routines for survival... \\ Helped me to thrive, so... \\ Despite \\ Failing and falling through the cracks \\ And no one ever investigating \\ I'm having to relearn again \\ Reading and writing, and being skills
}

\author{
Just another Invisible Disability \\ New vocabulary like \\ Executive functioning and \\ Comorbid and \\ Rejection Sensitivity and \\ Time-Blindness and \\ Motivation-less and \\ Adder*ll and \\ Cognitive Distortions and \\ Inattentive and \\ Emotional Dysregulation and \\ Working Memory and \\ Hyperactivity and
}


It's so much more than

I lost my keys

Or

I forget a lot

It's an invisible game

And I am the Jenga

\section{Just another Realization}

ADHD is the validation of my life

Before I was diagnosed

I owned $w^{*}$ ird, or brok* $n$, or *dd

But rather; I was invisible and ignored

Like many score before

Black girls in urban/poor/any/all schools

In the educational jungle

An educational ecosystem

With barely enough staff or resources

Become Black girls in liberal arts colleges

Referred to inaccessible private practice

For expensive testing

That didn't take Medicaid

Become Black girls in the Ivy League

Willing themselves to perform excellence

Because the ancestors...

\section{Just a Unicorn}

Become Black girls in Ph.D. programs

Finally learning how they learn

Even when it feels too hard

Even when the stigmas are real

Even when good island gyals don't...

Ask. For. Help.

Get. Diagnosed.

Take. Medication.

Use. Accommodations.

Write. About. It. Publicly.

Look for the healing in the message. 